\title{
Complete genome sequence of Brachybacterium faecium type strain (Schefferle 6-10 ${ }^{\top}$ )
}

\author{
Alla Lapidus ${ }^{1}$, Rüdiger Pukall ${ }^{2}$, Kurt LaButtii ${ }^{1}$, Alex Copeland ${ }^{1}$, Tijana Glavina Del Rio ${ }^{1}$, Matt $^{2}$ \\ Nolan', Feng Chen', Susan Lucas', Hope Tice ${ }^{1}$, Jan-Fang Cheng1, David Bruce ${ }^{1,3}$, Lynne \\ Goodwin ${ }^{1,3}$, Sam Pitluck1, Manfred Rohde ${ }^{4}$, Markus Göker², Amrita Pati ${ }^{1}$, Natalia Ivanova ${ }^{1}$, \\ Konstantinos Mavromatis ${ }^{1}$, Amy Chen ${ }^{5}$, Krishna Palaniappan ${ }^{5}$, Patrik D'haeseleer ${ }^{1,6}$, Patrick \\ Chain $^{1,6}$, Jim Bristow ${ }^{1}$, Jonathan A. Eisen ${ }^{1,7}$, Victor Markowitz ${ }^{5}$, Philip Hugenholtz ${ }^{1}$, Nikos C. \\ Kyrpides $^{1}$, and Hans-Peter Klenk ${ }^{2 *}$ \\ ${ }^{1}$ DOE Joint Genome Institute, Walnut Creek, California, USA \\ ${ }^{2}$ DSMZ - German Collection of Microorganisms and Cell Cultures GmbH, Braunschweig, \\ Germany \\ ${ }^{3}$ Los Alamos National Laboratory, Bioscience Division, Los Alamos, New Mexico, USA \\ ${ }^{4} \mathrm{HZI}$ - Helmholtz Centre for Infection Research, Braunschweig, Germany \\ ${ }^{5}$ Biological Data Management and Technology Center, Lawrence Berkeley National Labora- \\ tory, Berkeley, California, USA \\ ${ }^{6}$ Lawrence Livermore National Laboratory, Livermore, California, USA \\ ${ }^{7}$ University of California Davis Genome Center, Davis, California, USA
}

${ }^{*}$ Corresponding author: $\underline{\text { Hans-Peter Klenk }}$

Keywords: mesophile, free-living, non-pathogenic, aerobic, rod-coccus growth cycle, uric acid degradation, Dermabacteraceae

Brachybacterium faecium Collins et al. 1988 is the type species of the genus, and is of phylogenetic interest because of its location in the Dermabacteraceae, a rather isolated family within the actinobacterial suborder Micrococcineae. B. faecium is known for its rod-coccus growth cycle and the ability to degrade uric acid. It grows aerobically or weakly anaerobically. The strain described in this report is a free-living, nonmotile, Gram-positive bacterium, originally isolated from poultry deep litter. Here we describe the features of this organism, together with the complete genome sequence, and annotation. This is the first complete genome sequence of a member of the actinobacterial family Dermabacteraceae, and the 3,614,992 bp long single replicon genome with its 3129 protein-coding and 69 RNA genes is part of the Genomic Encyclopedia of Bacteria and Archaea project.

\section{Introduction}

Strain Schefferle 6-10 ${ }^{\mathrm{T}}$ (DSM $4810=$ ATCC 43885

$=$ JCM $11609=$ NCIMB 9860) is the type strain of Brachybacterium faecium, which is the type species of the genus Brachybacterium [1] (Figure 1). B. faecium was described by Collins et al. in 1988 [1] as Gram-positive and nonmotile. The organism is of significant interest for its position in the tree of life where the rapidly growing genus Brachybacterium (12 species) is located in the actinobacterial family Dermabacteraceae [2].
Only two accompanying strains (Schefferle 3-8 = NCIMB 9859, and Schefferle 7-11 = NCIMB 9861) were initially reported from the poultry deep litter from which the type strain Schefferle $6-10^{\mathrm{T}}$ was isolated [3]. Both strains were later reclassified as members of other Brachybacterium species: $B$. conglomeratum (NCIMB 9859) and B. paraconglomeratum (NCIMB 9861). Some closely related isolates with more than 98.5\% 16S rRNA gene sequence identity were reported from ma- 
rine sediments (JH107; FJ572028), deep-sea sediments (PB10; DQ643203), and living room air (Gauze_W_12_19; FJ267545). Only four sequences from uncultured bacteria are accessible via EMBL, showing at least $98 \%$ sequence identity to B. faecium. These sequences were derived from the analyses of urban aerosols (DQ129569), human vaginal epithelium (AY959187), floor dust (FM872846), and water $20 \mathrm{~m}$ downstream of manure (AY212613). No phylotypes from environmental screening or genomic surveys could be linked to B. faecium (as of February 2009). Here we present a summary classification and a set of features for B. faecium Schefferle $6-10^{\mathrm{T}}$ (Table 1), together with the description of the complete genomic sequencing and annotation.

\section{Classification and features}

B. faecium Schefferle 6-10 $10^{\mathrm{T}}$ cells vary in shape and exhibit a rod-coccus growth cycle, which is not atypical of this evolutionary group. Cells in the stationary phase are predominantly coccoid (Figure 2 ), whereas cells in fresh cultures are irregular, slender rods [1]. Cells are frequently arranged at an angle to give V-formations [1] (Figure 2). $B$. faecium cells are non-acid fast and do not form endospores [1]. B. faecium is essentially aerobic, but is also capable of very weak growth under anaerobic conditions [1].

B. faecium is capable of degrading uric acid, and fermenting cellobiose, glucose, maltose, and mannose, but not cellulose, chitin, or gelatin. The optimal growth temperature is $25-30^{\circ} \mathrm{C}$. Nitrate is reduced to nitrite by some $B$. faecium strains [1] as a candidate for terminal electron acceptor during anaerobic growth.

Figure 1 shows the phylogenetic neighborhood of B. faecium strain Schefferle $6-10^{\mathrm{T}}$ in a $16 \mathrm{~S}$ rRNA based tree. The sequences of the three 16S rRNA genes in the $B$. faecium Schefferle $6-10^{\mathrm{T}}$ genome differ by up to two nucleotides (nts) from each other, and by three nts from the reference sequence of strain DSM 4810 (X91032). The slight differences between the genome data and the previously reported $16 \mathrm{~S}$ rRNA gene sequence is most likely due to sequencing errors in the previously reported sequence data.

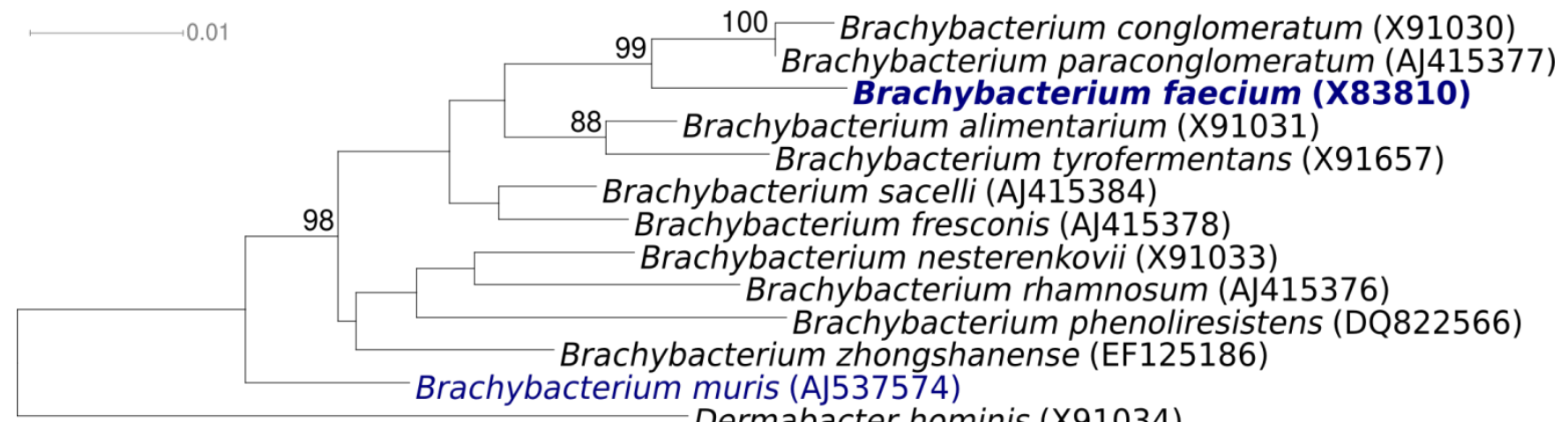

Dermabacter hominis (X91034)

Figure 1. Phylogenetic tree of B. faecium Schefferle $6-10^{\top}$ and all type strains of the genus Brachybacterium, inferred from 1408 aligned characters [4] of the 16S rRNA sequence under the maximum likelihood criterion [5,6]. The tree was rooted with Dermabacter hominis, another member of the family Dermabacteraceae. The branches are scaled in terms of the expected number of substitutions per site. Numbers above branches are support values from 1000 bootstrap replicates, if larger than $60 \%$. Strains with a genome-sequencing project registered in GOLD [7] are printed in blue; published genomes in bold. 


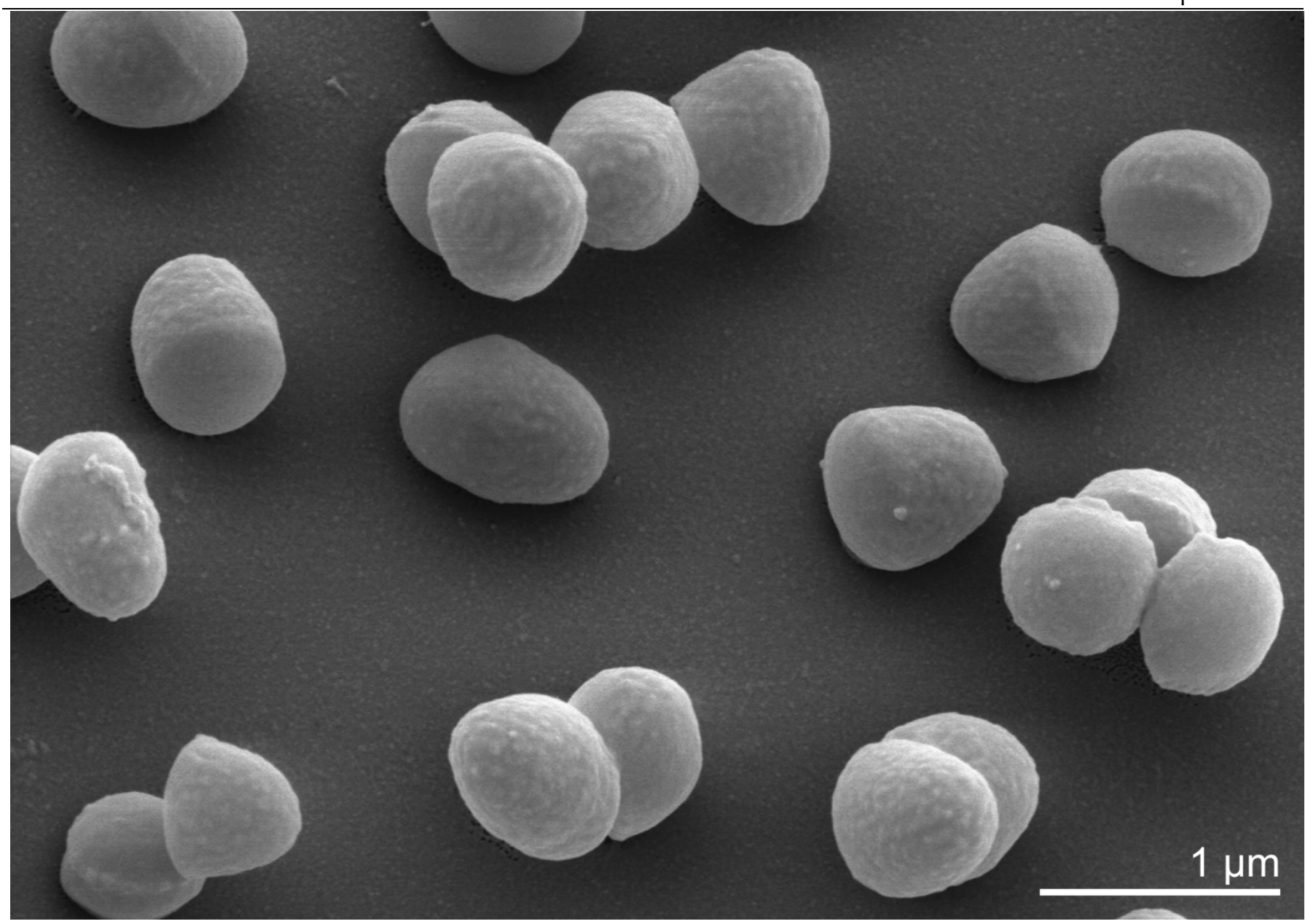

Figure 2. Scanning electron micrograph of B. faecium Schefferle 6-10

\section{Chemotaxonomy}

Strain Schefferle 6-10 $10^{\mathrm{T}}$ was originally described as a coryneform bacterium. This descriptive term applies to a diverse range of taxa and indicates that the comparisons made in the original publication need to be reviewed. The murein of $B$. faecium contains meso-diaminopimelic acid, alanine and glutamic acid. The strain possesses a type $\mathrm{A} 4 \gamma$ peptidoglycan, type A31.2 according to the German Collection of Microorganisms and Cell Cultu-

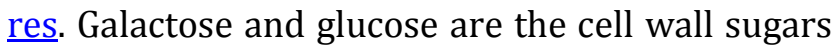
[1]. As in other Brachybacterium strains, the fatty acid pattern of strain Schefferle $6-10^{\mathrm{T}}$ is dominated by branched-chain saturated anteiso- (ai-) fatty acids: ai- $\mathrm{C}_{15: 0}(40 \%)$, ai- $\mathrm{C}_{17: 0}(37 \%)$, and $\mathrm{C}_{16: 0}$ and iso- $\mathrm{C}_{16: 0} 7.5 \%$, each, with smaller amounts of iso$\mathrm{C}_{15: 0}(3.5 \%)$, iso- $\mathrm{C}_{17: 0}(2.0 \%)$ [1]. Straight chain and unsaturated fatty acids are absent [1]. As usual for most members of the Actinomycetales, mycolic acids were not reported [1]. A menaqui- none with seven isoprene units (MK-7) predominates (88\%) complemented by $11 \%$ MK-8 [1]. Phosphatidylglycerol and diphosphatidylglycerol were identified as the dominant polar lipids, together with several glycolipids and an unknown phospholipid [1]. The $\mathrm{R}_{\mathrm{f}}$ values of the glycolipids suggest that they contain different numbers of sugars (one, two or possibly three) and may also show differences in the nature and linkage of the sugars. It is not known whether these glycolipids are based on a diglyceride or whether they contain an acylated sugar, directly linked to a monoglyceride. The chemical composition is typical of members of the genus Brachybacterium and similar, but not identical with the members of the only other genus placed in the family Dermabacteraceae, Dermabacter. In addition to cytochrome aa3, B. faecium possesses cytochrome $\mathrm{d}_{626}$, and cytochrome $c_{550}[8]$. 
Table 1. Classification and general features of B. faecium Schefferle $6-10^{\top}$ based on MIGS recommendations [9]

\begin{tabular}{|c|c|c|c|}
\hline MIGS ID & Property & Term & $\begin{array}{l}\text { Evidence } \\
\text { code }\end{array}$ \\
\hline & Current classification & $\begin{array}{ll}\text { Domain } & \text { Bacteria } \\
\text { Phylum } & \text { Actinobacteria } \\
\text { Class } & \text { Actinobacteria } \\
\text { Order } & \text { Actinomycetales } \\
\text { Family } & \text { Dermabacteraceae } \\
\text { Genus } & \text { Brachybacterium } \\
\text { Species } & \text { Brachybacterium faecium } \\
\text { Type strain } & \text { Schefferle 6-10 }\end{array}$ & $\begin{array}{l}\text { TAS [2] } \\
\text { TAS [2] } \\
\text { TAS [2] } \\
\text { TAS [1] } \\
\text { TAS [1] }\end{array}$ \\
\hline & Gram stain & positive & TAS [1] \\
\hline & Cell shape & varies; rod-coccus growth cycle & TAS [1] \\
\hline & Motility & nonmotile & TAS [1] \\
\hline & Sporulation & non-sporulating & TAS [1] \\
\hline & Temperature range & mesophilic & TAS [1] \\
\hline & Optimum temperature & $25-30^{\circ} \mathrm{C}$ & TAS [1] \\
\hline & Salinity & $5 \mathrm{~g} \mathrm{NaCl} / \mathrm{L}$ & TAS [1] \\
\hline \multirow[t]{3}{*}{ MIGS-22 } & Oxygen requirement & $\begin{array}{l}\text { aerobic; very weak growth under } \\
\text { anaerobic conditions }\end{array}$ & TAS [1] \\
\hline & Carbon source & glucose, maltose, mannose, cellobiose & TAS [1] \\
\hline & Energy source & starch & NAS \\
\hline MIGS-6 & Habitat & deep litter (soil) & TAS [3] \\
\hline MIGS-15 & Biotic relationship & free-living & NAS \\
\hline \multirow[t]{3}{*}{ MIGS-14 } & Pathogenicity & none & NAS \\
\hline & Biosafety level & 1 & TAS [10] \\
\hline & Isolation & poultry deep litter & TAS [3] \\
\hline MIGS-4 & Geographic location & & TAS [3] \\
\hline MIGS-5 & Sample collection time & about 1966 & TAS [3] \\
\hline $\begin{array}{l}\text { MIGS-4.1 } \\
\text { MIGS-4.2 }\end{array}$ & Latitude - Longitude & not reported & \\
\hline MIGS-4.3 & Depth & not reported & \\
\hline MIGS-4.4 & Altitude & not reported & \\
\hline
\end{tabular}

Evidence codes - IDA: Inferred from Direct Assay (first time in publication); TAS: Traceable Author Statement (i.e., a direct report exists in the literature); NAS: Non-traceable Author Statement (i.e., not directly observed for the living, isolated sample, but based on a generally accepted property for the species, or anecdotal evidence). These evidence codes are from the Gene Ontology project [11]. If the evidence code is IDA, then the property should have been directly observed, for the purpose of this specific publication, for a live isolate by one of the authors, or an expert or reputable institution mentioned in the acknowledgements.

\section{Genome sequencing and annotation Genome project history}

This organism was selected for sequencing on the basis of its phylogenetic position, and is part of the Genomic Encyclopedia of Bacteria and Archaea project. The genome project is deposited in the Genomes OnLine Database [7] and the complete genome sequence in GenBank (CP001643). Sequencing, finishing and annotation were performed by the DOE Joint Genome Institute (JGI). A summary of the project information is shown in Table 2 . 


\begin{tabular}{lll}
\hline Table 2. Genome sequencing project information & Term \\
\hline MIGS ID & Property & Finished \\
\hline MIGS-31 & Finishing quality & Two genomic libraries: 8kb pMCL200 \\
& & and fosmid pcc1Fos Sanger libraries \\
MIGS-28 & Libraries used & One 454 pyrosequence standard library \\
& & and one Illumina library \\
MIGS-29 & Sequencing platforms & ABI3730, 454 GS FLX, Illumina GA \\
MIGS-31.2 & Sequencing coverage & $10 x$ Sanger; 40x pyrosequence \\
MIGS-30 & Assemblers & Newbler version 1.1.02.15, PGA \\
MIGS-32 & Gene calling method & Genemark 4.6b, tRNAScan-SE-1.23, in- \\
& Genbank ID & CP001643 \\
& Genbank Date of Release & N/A \\
& GOLD ID & $\underline{\text { Gi02066 }}$ \\
& NCBI project ID & $\underline{17026}$ \\
& Database: IMG-GEBA & $\underline{2500868055}$ \\
& Source Material Identifier & DSM 4810 \\
MIGS-13 & Project relevance & Tree of Life, GEBA \\
\hline
\end{tabular}

\section{Growth conditions and DNA isolation}

B. faecium Schefferle 6-10 ${ }^{\mathrm{T}}$, DSM 4810, was grown in DSMZ medium 92 (with $3 \%$ trypticase soy broth, $0.3 \%$ yeast extract) at $28^{\circ} \mathrm{C}$. DNA was isolated from 1-1.5 $\mathrm{g}$ of cell paste using Qiagen Genomic 500 DNA Kit (Qiagen, Hilden, Germany) without modification of the manufacturer's protocol for cell lysis.

\section{Genome sequencing and assembly}

The genome was sequenced using a combination of Sanger, 454 and Illumina sequencing platforms. All general aspects of library construction and sequencing performed at the JGI can be found on the JGI website. 454 Pyrosequencing reads were assembled using the Newbler assembler version 1.1.02.15 (Roche). Large Newbler contigs were broken into 4,074 overlapping fragments of 1,000 bp and entered into the assembly as pseudo-reads. The sequences were assigned quality scores based on Newbler consensus q-scores with modifications to account for overlap redundancy and to adjust inflated q-scores. A hybrid 454/Sanger assembly was made using the PGA assembler. Possible mis-assemblies were corrected and gaps between contigs were closed by custom primer walks from sub-clones or PCR products. 258 Sanger finishing reads were produced. Illumina reads were used to improve the final consensus quality using an in-house developed tool (the Polisher). The error rate of the completed genome sequence is less than 1 in 100,000. Together all sequence types provided $50 \mathrm{x}$ coverage of the genome.

\section{Genome annotation}

Genes were identified using GeneMark [12] as part of the genome annotation pipeline in the Integrated Microbial Genomes Expert Review (IMGER) system [13], followed by a round of manual curation using the JGI GenePRIMP pipeline [14]. The predicted CDSs were translated and used to search the National Center for Biotechnology Information (NCBI) nonredundant database, UniProt, TIGRFam, Pfam, PRIAM, KEGG, COG, and InterPro databases. The tRNAScanSE tool [15] was used to find tRNA genes, whereas ribosomal RNAs were found by using the tool RNAmmer [16]. Other non-coding RNAs were identified by searching the genome for the Rfam profiles using INFERNAL (v0.81) [17]. Additional gene prediction analysis and manual functional annotation was performed within the Integrated Microbial Genomes (IMG) platform [18].

\section{Metabolic network analysis}

The metabolic Pathway/Genome Database (PGDB) was computationally generated using Pathway Tools software version 12.5 [19] and MetaCyc version 12.5 [20], based on annotated EC numbers and a customized enzyme name mapping file. It has undergone no subsequent manual curation and may contain errors, similar to a Tier 3 BioCyc PGDB [21].

\section{Genome properties}

The genome is 3,614,992 bp long and comprises one circular chromosome with a $72.1 \%$ GC con- 
tent (Table 3 and Figure 3). Of the 3,198 genes predicted, 3,129 were protein coding genes, and 69 RNAs. Sixty pseudogenes were also identified. The majority of genes $(77.3 \%)$ of the genes were assigned with a putative function while the remaining ones are annotated as hypothetical pro- teins. The properties and the statistics of the genome are summarized in Table 3. The distribution of genes into COGs functional categories is presented in Table 4, and a cellular overview diagram is presented in Figure 4, followed by a summary of metabolic network statistics shown in Table 5.

Table 3. Genome Statistics

\begin{tabular}{lrr}
\hline Attribute & Value & \% of Total \\
\hline Genome size (bp) & $3,614,992$ & \\
DNA Coding region (bp) & $3,287,735$ & $90.95 \%$ \\
DNA G+C content (bp) & $2,604,449$ & $72.05 \%$ \\
Number of replicons & 1 & \\
Extrachromosomal elements & 0 & \\
Total genes & 3198 & \\
RNA genes & 69 & $2.16 \%$ \\
rRNA operons & 3 & \\
Protein-coding genes & 3129 & $97.84 \%$ \\
Pseudo genes & 60 & $1.88 \%$ \\
Genes with function prediction & 2473 & $77.33 \%$ \\
Genes in paralog clusters & 347 & $10.85 \%$ \\
Genes assigned to COGs & 2371 & $74.14 \%$ \\
Genes assigned Pfam domains & 2440 & $76.30 \%$ \\
Genes with signal peptides & 697 & $21.79 \%$ \\
Genes with transmembrane helices & 836 & $26.14 \%$ \\
CRISPR repeats & 0 & \\
\hline
\end{tabular}

Table 4. Number of genes associated with the 21 general COG functional categories

\begin{tabular}{lrrl} 
Code & Value & \% & Description \\
\hline J & 163 & 5.2 & Translation, ribosomal structure and biogenesis \\
A & 1 & 0.0 & RNA processing and modification \\
K & 204 & 6.5 & Transcription \\
L & 125 & 4.0 & Replication, recombination and repair \\
B & 1 & 0.0 & Chromatin structure and dynamics \\
D & 19 & 0.6 & Cell cycle control, mitosis and meiosis \\
Y & 0 & 0.0 & Nuclear structure \\
V & 53 & 1.7 & Defense mechanisms \\
T & 88 & 2.8 & Signal transduction mechanisms \\
M & 127 & 4.1 & Cell wall/membrane biogenesis \\
N & 2 & 0.1 & Cell motility \\
Z & 1 & 0.0 & Cytoskeleton \\
W & 0 & 0.0 & Extracellular structures \\
U & 25 & 0.8 & Intracellular trafficking and secretion \\
O & 68 & 2.2 & Posttranslational modification, protein turnover, chaperones \\
C & 136 & 4.3 & Energy production and conversion \\
G & 366 & 11.7 & Carbohydrate transport and metabolism \\
\hline
\end{tabular}


Table 4. Number of genes associated with the 21 general COG functional categories (cont.)

\begin{tabular}{lrrl} 
Code & \multicolumn{1}{c}{ Value } & \% & Description \\
\hline E & 261 & 8.3 & Amino acid transport and metabolism \\
F & 84 & 2.7 & Nucleotide transport and metabolism \\
H & 107 & 3.4 & Coenzyme transport and metabolism \\
I & 92 & 2.9 & Lipid transport and metabolism \\
P & 145 & 4.6 & Inorganic ion transport and metabolism \\
Q & 44 & 1.4 & Secondary metabolites biosynthesis, transport and catabolism \\
R & 333 & 10.6 & General function prediction only \\
S & 189 & 6.0 & Function unknown \\
- & 758 & 24.2 & Not in COGs \\
\hline
\end{tabular}

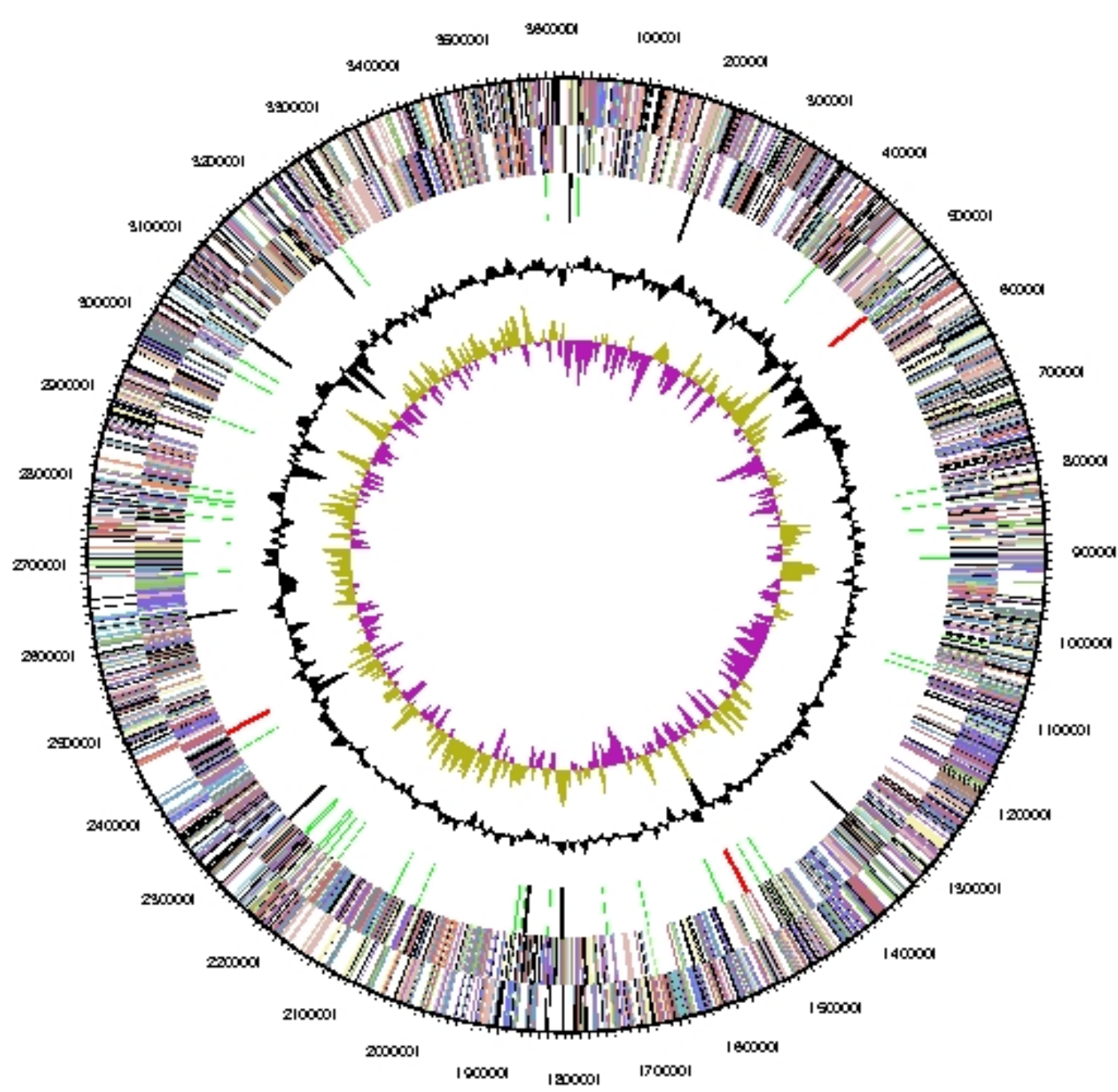

Figure 3. Graphical circular map of the genome. From outside to the center: Genes on forward strand (color by COG categories), Genes on reverse strand (color by COG categories), RNA genes (tRNAs green, rRNAs red, other RNAs black), GC content, GC skew. 


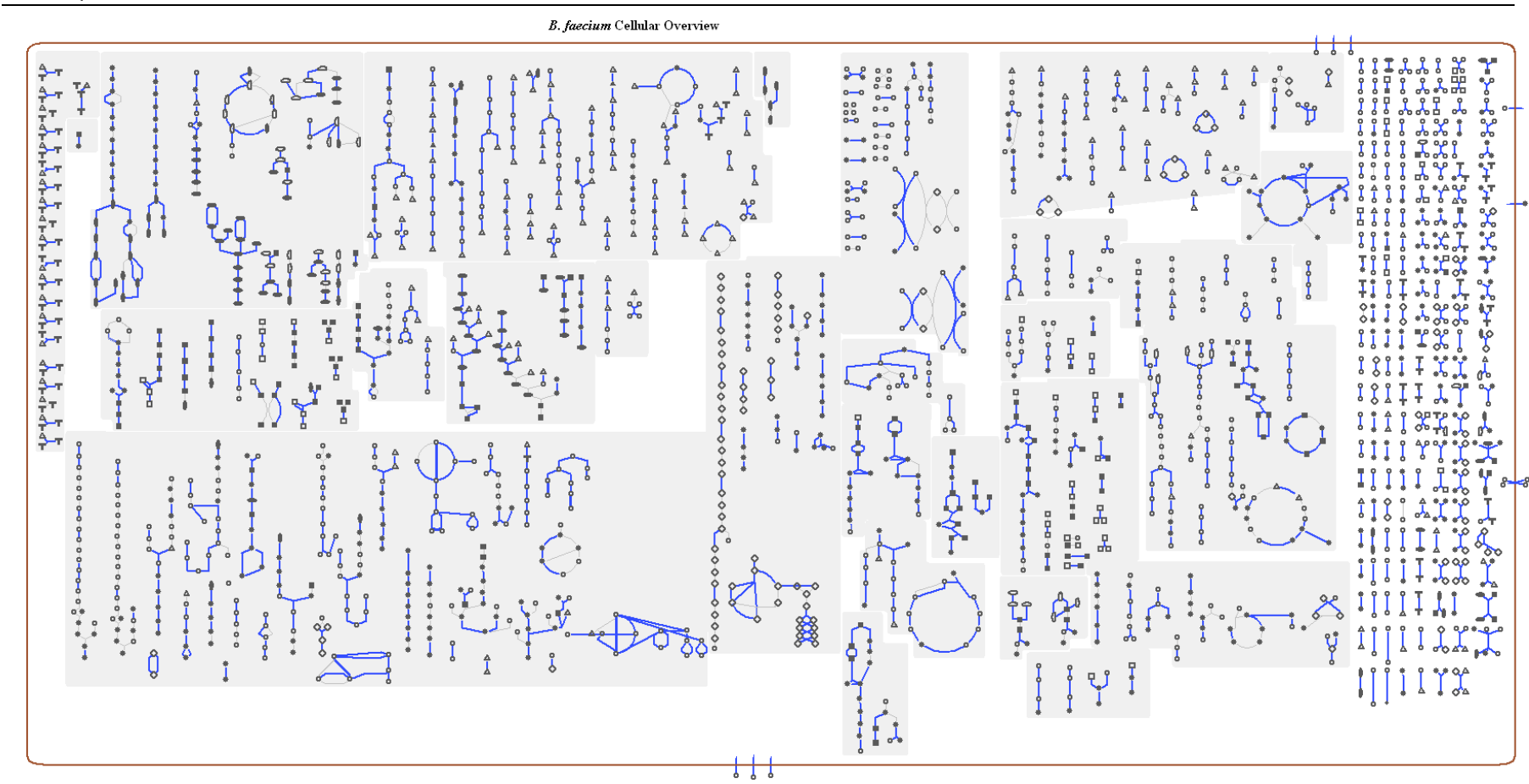

Figure 4. Schematic cellular overview of all pathways of the B. faecium strain Schefferle 6-10 ${ }^{\mathrm{T}}$ metabolism. Nodes represent metabolites, with shape indicating class of metabolite. Lines represent reactions.

Table 5. Metabolic Network Statistics

\begin{tabular}{lr}
\hline Attribute & \multicolumn{1}{c}{ Value } \\
\hline Total genes & 3198 \\
Enzymes & 674 \\
Enzymatic reactions & 1031 \\
Metabolic pathways & 218 \\
Metabolites & 758 \\
\hline
\end{tabular}

\section{References}

1. Collins M, Brown J, Jones D. Brachybacterium faecium gen. nov., sp. nov., a coryneform bacterium from poultry deep litter. Int J Syst Bacteriol 1988; 38:45-48 doi:10.1099/00207713-38-1-45

2. Stackebrandt E, Rainey F, Ward-Rainey N. Proposal for a new hierarchic classification system, Actinobacteria classis nov. Int I Syst Bacteriol 1997; 47:479-491 doi:10.1099/00207713-47-2-479

3. Schefferle $\mathrm{H}$. Coryneform bacteria in poultry deep litter. I Appl Bacteriol 1966; 29:147-160 doi:10.1111/j.1365-2672.1966.tb03462.x

\section{Acknowledgements}

We gratefully acknowledge the help of Gabriele Gehrich-Schröter for growing B. faecium cultures and Susanne Schneider for DNA extraction and quality analysis (both at DSMZ). This work was performed under the auspices of the US Department of Energy's Office of Science, Biological and Environmental Research Program, and by the University of California, Lawrence Berkeley National Laboratory under contract No. DEAC02-05CH11231, Lawrence Livermore National Laboratory under Contract No. DE-AC52-07NA27344, and Los Alamos National Laboratory under contract No. DEAC02-06NA25396, as well as German Research Foundation (DFG) INST 599/1-1.

4. Lee C, Grasso C, Sharlow MF. Multiple sequence alignment using partial order graphs. Bioinformatics 2002; 18:452-464 PMID:11934745 $\underline{\text { doi:10.1093/bioinformatics/18.3.452 }}$

5. Felsenstein J. Evolutionary trees from DNA sequences: a maximum likelihood approach. J Mol Evol 1981; 17:368-376 PMID:7288891 doi:10.1007/BF01734359

6. Stamatakis A, Hoover P, Rougemont J. A rapid bootstrap algorithm for the RAxML Web servers. Syst Biol 2008; 57:758-771 PMID:18853362 doi:10.1080/10635150802429642 
Lapidus et al.

7. Liolios K, Mavromatis K, Tavernarakis N, Kyrpides NC. The Genomes On Line Database (GOLD) in 2007: status of genomic and metagenomic projects and their associated metadata. Nucleic Acids Res 2008; 36:D475479 PMID:17981842 doi:10.1093/nar/gkm884

8. Schleifer K, Lang K. Close relationship among strains of Micrococcus conglomeratus and Arthrobacter sp. FEMS Microbiol 1980; 9:223226 doi:10.1111/j.1574-6968.1980.tb05641.x

9. Field D, Garrity G, Gray T, Morrison N, Selengut J, Sterk P, Tatusova T, Thomson N, Allen MJ, Angiuoli SV, et al. The minimum information about a genome sequence (MIGS) specification. Nat Biotechnol 2008; 26:541547 PMID:18464787 doi:10.1038/nbt1360

10. Anonymous. Biological Agents: Technical rules for biological agents. <www.baua.de $>$.

11. Ashburner M, Ball CA, Blake JA, Botstein D, Butler H, Cherry JM, Davis AP, Dolinski K, Dwight SS, Eppig JT, et al. Gene ontology: tool for the unification of biology. The Gene Ontology Consortium. Nat Genet 2000; 25:25-29 PMID:10802651 doi:10.1038/75556

12. Besemer J, Lomsadze A, Borodovsky M. GeneMarkS: a self-training method for prediction of gene starts in microbial genomes. Implications for finding sequence motifs in regulatory regions. Nucleic Acids Res 2001; 29:2607-2618 PMID:11410670 doi:10.1093/nar/29.12.2607

13. Markowitz V, Mavromatis K, Ivanova N, Chen I-M, Chu K, Palaniappan K, Szeto E, Anderson I, Lykidis A, Kyrpides N. Expert Review of Functional Annotations for Microbial Genomes. Bioinformatics, 2009; in press.

14. Pati A. GenePRIMP: A Gene Prediction Improvement Pipeline for microbial genomes (In preparation 2009).
15. Lowe TM, Eddy SR. tRNAscan-SE: a program for improved detection of transfer RNA genes in genomic sequence. Nucleic Acids Res 1997; 25:955-964 PMID:9023104 doi:10.1093/nar/25.5.955

16. Lagesen K, Hallin P, Rodland EA, Staerfeldt $\mathrm{HH}$, Rognes T, Ussery DW. RNAmmer: consistent and rapid annotation of ribosomal RNA genes. Nucleic Acids Res 2007; 35:31003108 PMID:17452365 doi:10.1093/nar/gkm160

17. Griffiths-Jones S, Moxon S, Marshall M, Khanna A, Eddy SR, Bateman A. Rfam: annotating non-coding RNAs in complete genomes. Nucleic Acids Res 2005; 33:D121D124 PMID:15608160 doi:10.1093/nar/gki081

18. Markowitz VM, Szeto E, Palaniappan K, Grechkin Y, Chu K, Chen IM, Dubchak I, Anderson I, Lykidis A, Mavromatis K, et al. The integrated microbial genomes (IMG) system in 2007: data content and analysis tool extensions. Nucleic Acids Res 2008; 36:D528D533 PMID:17933782 doi:10.1093/nar/gkm846

19. Karp P, Paley S, Romero P. The pathway tools software. Bioinformatics 2000; 18:S225-232

20. Caspi R, Foerster H, Fulcher CA, Kaipa P, Krummenacker $M$, Latendresse $M$, Paley $S$, Rhee SY, Shearer AG, Tissier C, et al. The MetaCyc Database of metabolic pathways and enzymes and the BioCyc collection of Pathway/Genome Databases. Nucleic Acids Res 2008; 36:D623-631 PMID:17965431 doi:10.1093/nar/gkm900

21. Karp PD, Ouzounis CA, Moore-Kochlacs C, Goldovsky L, Kaipa P, Ahren D, Tsoka S, Darzentas N, Kunin V, Lopez-Bigas N. Expansion of the BioCyc collection of pathway/genome databases to 160 genomes. Nucleic Acids Res 2005; 33:6083-6089 PMID:16246909 doi:10.1093/nar/gki892 